Pacific Journal of Mathematics

UNIVERSAL INTERPOLATING SETS AND THE
NEVANLINNA-PICK PROPERTY IN BANACH SPACES O

A. K. SNYDER 


\section{UNIVERSAL INTERPOLATING SETS AND THE NEVANLINNA-PICK PROPERTY IN BANACH SPACES OF FUNCTIONS}

\section{A. K. SNYDER}

1. Introduction. Let $E$ be a Banach space of functions on $S, W \subset S$, and let $M(E)$ be the multiplier algebra of $E$. Consider the restriction space $E \mid W$ as a quotient of $E$. The space $E$ has the Nevanlinna-Pick property relative to $W$ if $M(E \mid W)=M(E) \mid W$ isometrically; $E$ has the factorization property relative to $W$ if there exists $u \in M(E)$ such that $u$ is an isometry of $E \mid W$ onto the annihilator of $S / W$ in $E$. We consider the problem of characterizing those spaces with the Nevanlinna-Pick property.

Theorem 1 solves this problem for suitable sequence spaces. It is shown that the Nevanlinna-Pick property of $E$ is equivalent to a natural factorization property of annihilators in the series space of $E$. It follows that $E$ has the Nevanlinna-Pick property relative to $W$ whenever $M(E)$ has the factorization property relative to $W$. A technique is provided in Lemma 6 for applying these sequence space results to general Banach spaces of functions. An identification of the dual of $H^{2} \mid W$ yields a proof of the classical Nevanlinna-Pick theorem based solely on the elementary factorization theory of the Hardy spaces. Zero set considerations yield the failure of the Nevanlinna Pick theorem in the Bergman spaces.

Applications are given to universal interpolating set problems in general Banach spaces of functions. Let $l^{2}(S)$ be the usual Hilbert space of functions on $S$ where $S$ has counting measure. Let $H$ be a Hilbert space of functions on $S$. A subset $W$ of $S$ is a universal interpolating set for $H$ if there exists a multiplier from $H \mid W$ onto $l^{2}(W)$. We show that $W$ is a universal interpolating set for $H$ if and only if $M(H \mid W)=l^{\infty}(W)$, the space of bounded functions on $W$. This result provides a convenient definition of universal interpolating sets for general Banach spaces of functions. It follows that if $E$ and $F$ are Banach spaces of functions on $S, M(E) \subset M(F), W$ is a universal interpolating set for $E$, and $E$ has the Nevanlinna-Pick property relative to $W$, then $W$ is a universal interpolating set for $F$. These results provide generalizations of some theorems of Shapiro and Shields on weighted interpolation in the Hardy space $H^{2}$ and the Bergman space $A^{2}$.

Finally, it is shown under weak assumptions that universal interpolating sequences always exist for Hilbert spaces of functions but may fail to exist for Banach spaces of functions. 
2. The series space. Let $S$ be a set and let $E$ be a family of complex-valued functions on $S$ which is a linear space under the pointwise operations. For each $s \in S$ let $\pi^{s}(f)=f(s), f \in E$. If $E$ is a Banach space such that each $\pi^{s}$ is continuous on $E$, then $E$ is called a Banach space of functions on $S$. The multiplier algebra $M(E)$ of $E$ is the family of complex-valued functions $u$ on $S$ such that $u f \in$ $E$ for all $f \in E$, the multiplication being pointwise. By the closed graph theorem each such $u$ acts as a bounded operator on the Banach space $E$ of functions. In case $\pi^{s} \neq 0$ on $E$ for each $s \in S$, then $M(E)$ is a Banach space of functions on $S$ with the operator norm.

Let $E$ be a Banach space of functions on $S$. For each $s \in S$ let $e^{s}$ be the function defined by $e^{s}(t)=0$ for $t \neq s, e^{s}(s)=1$. Let $e(s)=1$ for all $s$. Let $\phi$ denote the linear span of $\left\{e^{s}: s \in S\right\}$. If $\phi \subset E$ let the functional dual $E^{f}$ of $E$ be the family of functions $g$ on $S$ given by $g(s)=F\left(e^{s}\right)$ for $F$ in the dual $E^{*}$ of $E$. If $\varphi$ is dense in $E$ then $E^{f}$ may be identified with $E^{*}$. Thus, $E^{f}$ may be considered as a Banach space of functions on $S$ with the dual space norm of $E^{*}$. If $S$ is a countable set, then $E$ is called a $B K$ space.

Throughout this work assume that $M(E)$ and $E^{f}$ have the norms as given above.

Let $E$ and $F$ be Banach spaces of functions on $S$ with $\varphi$ dense in $E$ and $F$. It is easy to see that $E=F$ isometrically if and only if $E^{f}=F^{f}$ isometrically.

If $E$ and $F$ are Banach spaces of functions on $S$ let $E \otimes F$ denote the set of all functions $u$ on $S$ of the form $u=\sum_{n} x^{n} y^{n}$ where $x^{n} \in$ $E, y^{n} \in F$, and $\sum_{n}\left\|x^{n}\right\|_{E}\left\|y^{n}\right\|_{F}<\infty$. Let $E \otimes F$ have the norm given by

$$
\|u\|_{E \otimes F}=\inf \left\{\sum_{n}\left\|x^{n}\right\|_{E}\left\|y^{n}\right\|_{F}: x^{n} \in E, y^{n} \in F, u=\sum_{n} x^{n} y^{n}\right\}
$$

Then $E \otimes F$ is the diagonal restriction of the projective tensor product of $E$ and $F$.

Let $E$ be a $B K$ space in which $\phi$ is dense. The series space $\mathscr{S}(E)$ of $E$ consists of all functions $u$ of the form $u=\sum_{n} x^{n} y^{n}$ where $x^{n} \in \varphi, y^{n} \in E^{f}$, and $\sum_{n}\left\|x^{n}\right\|_{E}\left\|y^{n}\right\|_{E^{f_{i}}}<\infty$. For $u \in \mathscr{S}(E)$ let

$$
\|u\|=\inf \left\{\sum_{n}\left\|x^{n}\right\|_{E}\left\|y^{n}\right\|_{E^{f}}: x^{n} \in \varphi, y^{n} \in E^{f}, u=\sum_{n} x^{n} y^{n}\right\} .
$$

It follows that $\mathscr{S}(E)$ is a $B K$ space with the above norm and that $\varphi$ is dense in $\mathscr{S}(E)$. Also, $\mathscr{S}(E)=E \otimes E^{f}$ isometrically. See for instance [3] for a discussion of the series space. It is known that $\mathscr{S}(E)^{f} \subset M(E) \subset M(\mathscr{S}(E))$.

The $B K$ space $E$ in which $\varphi$ is dense is strongly series summable. if there exists $\left\{u^{n}\right\} \subset \varnothing$ such that $\lim _{n} u^{n}(s)=1$ for each $s$ and $\left\{u^{n}\right\}$ 
is bounded in $M(E) . \quad E$ is series summable if $\mathscr{S}(E)^{f}=M(E)$ isometrically.

It is known that $E$ is series summable if and only if $e \in \mathscr{S}(E)^{f}$. Furthermore, $E$ is series summable if it is strongly series summable. See [3] for details.

3. Characterization of the Nevanlinna-Pick property. Let $E$ be a Banach space of functions on $S, W \subset S$. For $f \in E$ let $f \mid W$ denote the restriction of the function $f$ to $W$, and let $E \mid W=\{f \mid W$ : $f \in E\}$. The kernel of the restriction map is $W^{\perp}=\{f \in E: f(w)=0$ for all $w \in W\}$. Therefore, the restriction map of $E$ onto $E \mid W$ induces an isomorphism of the quotient $E / W^{\perp}$ onto $E \mid W$. Also, $W^{\perp}$ is a closed subspace of $E$ since the point evaluations are continuous. Hence, $E \mid W$ becomes a Banach space of functions on $W$ under the quotient norm of $E / W^{\perp}$. Specifically, for functions $g$ in $E \mid W$ let $\|g\|=$ $\inf \{\|f\|: f \mid W=g\}$. Assume throughout that restrictions of Banach spaces of functions have this particular norm.

Assume throughout this section that the equation $E=F$ for Banach spaces of functions includes the requirement that the norms coincide.

Lemma 1. Let $E$ be a $B K$ space on the set $S, W \subset S, \varphi$ dense in $E$. Let $K$ be the annihilator of $S \backslash W$ in $E$, and let $K^{\prime}$ be the annihilator of $S \backslash W$ in $E^{f}$. Then

(i ) $(K \mid W)^{f}=E^{f} \mid W$ if $\varphi \mid W$ is dense in $K \mid W$;

(ii) $(E \mid W)^{f}=K^{\prime} \mid W$ if $\varphi \mid S \backslash W$ is dense in $W^{\perp} \mid S \backslash W$.

Proof. (i) Note that by the Hahn-Banach theorem $G \in K^{*}$ if and only if $G$ has an extension to a member of $E^{*}$ and $\|G\|_{K^{*}}=$ $\inf \left\{\left\|G^{\wedge}\right\|_{E^{*}}: G^{\wedge}=G\right.$ on $\left.K\right\}$. But $g \in(K \mid W)^{f}$ if and only if there exists $G \in K^{*}$ with $g(s)=G\left(e^{s}\right), s \in W$. Similarly, $h \in E^{f} \mid W$ if and only if there exists $H \in E^{*}$ such that $h(s)=H\left(e^{8}\right), s \in W$. It follows that $(K \mid W)^{f}=E^{f} \mid W$.

(ii) Note that $(E \mid W)^{*}$ may be identified as the annihilator in $E^{*}$ of $W^{\perp}=\{f \in E: f \mid W=0\}$. But then $g \in(E \mid W)^{f}$ if and only if there exists $G \in E^{*}, G=0$ on $W^{\perp}$, such that $g(s)=G\left(e^{s}\right), s \in W$. However, $G=0$ on $W^{\perp}$ if and only if $G\left(e^{s}\right)=0, s \notin W$. This proves that $(E \mid W)^{f}=K^{\prime} \mid W$.

An elementary calculation involving the definitions of $E \mid W$ and $E \otimes F$ establishes the following.

LEMma 2. If $E$ and $F$ are Banach spaces of functions on $S$ and $W \subset S$, then $(E \otimes F) \mid W=(E \mid W) \otimes(F \mid W)$. 
Lemma 3. Let $E$ be a $B K$ space on $S, W \subset S$. If $E$ is series summable, then so is $E \mid W$. in $E$.

Proof. Note first that $\phi \mid W$ is dense in $E \mid W$ since $\varphi$ is dense

By Lemma $1,(E \mid W)^{f}=K^{\prime} \mid W$ where $K^{\prime}$ is the annihilator of $S \backslash W$ in $E^{f}$. Therefore, using Lemma 2, $\mathscr{S}(E \mid W)=E \mid W \otimes(E \mid W)^{f}=$ $E\left|W \otimes K^{\prime}\right| W=\left(E \otimes K^{\prime}\right) \mid W$. Thus, $\mathscr{S}(E \mid W)$ may be considered as a subspace of $\mathscr{S}(E)$ where members of $\mathscr{S}(E \mid W)$ vanish off $W$. Also, it follows that for $u \in \mathscr{S}(E \mid W),\|u\|_{\mathscr{S}(E \mid W)}=\|u\|_{\left(E \otimes K^{\prime}\right) \mid W}=\|u\|_{E \otimes K^{\prime}} \geqq$ $\|u\|_{E \otimes E^{f}}=\|u\|_{\mathscr{S}(E)}$.

Now let $u \in \varnothing$ with $\|u\|_{\mathscr{S}(E \mid W)} \leqq 1$. Then $\|u\|_{\mathscr{S}(E)} \leqq 1$. Therefore, $\left|\sum_{s \in W} u(s)\right| \leqq\|e\|_{\mathscr{S}(E)}$. It follows that $e \in \mathscr{S}(E \mid W)^{f}$, so $E \mid W$ is series summable.

Recall that $E$ has the Nevanlinna-Pick property relative to $W$ if $M(E \mid W)=M(E) \mid W$. Note that this definition differs from that given in [8]. The two definitions coincide in case $E$ is a dual space with weak-star continuous point evaluations. See [8], Theorem 3.

THEOREM 1. Let $E$ be a $B K$ space on $S, W \subset S$, and assume that $E$ is series summable. Let $K^{\prime}$ and $K^{\prime \prime}$ be the annihilators of $S \backslash W$ in $E^{f}$ and $\mathscr{S}(E)$, respectively. Then $E$ has the NevanlinnaPick property relative to $W$ if and only if $K^{\prime \prime}=E \otimes K^{\prime}$.

Proof. According to [3], 6.5(b), $\varphi \mid W$ is dense in $K^{\prime \prime} \mid W$, since $\mathscr{S}(E)$ must also be series summable. By Lemma 1, $M(E) \mid W=$ $\mathscr{S}(E)^{f} \mid W=\left(K^{\prime \prime} \mid W\right)^{f}$. Also, $M(E \mid W)=\mathscr{S}(E \mid W)^{f}$ using Lemma 3.

Since $K^{\prime \prime}$ and $E \otimes K^{\prime}$ vanish off $W$, the condition $K^{\prime \prime}=E \otimes K^{\prime}$ is equivalent to $K^{\prime \prime}\left|W=E \otimes K^{\prime}\right| W$. By Lemmas 1 and 2, the latter is equivalent to $K^{\prime \prime}|W=E| W \otimes(E \mid W)^{f}=\mathscr{S}(E \mid W)$. Therefore, $K^{\prime \prime}=E \otimes K^{\prime}$ if and only if $\left(K^{\prime \prime} \mid W\right)^{f}=\mathscr{S}(E \mid W)^{f}$, i.e., $M(E) \mid W=$ $M(E \mid W)$, i.e., $E$ has the Nevanlinna-Pick property relative to $W$.

Let $E$ be a Banach space of functions on $S$ and $u$ be a complexvalued function on $S, u(s) \neq 0$ for all $s \in S$. Let $u E=\{u x: x \in E\}$. Then $u E$ is a Banach space of functions on $S$ under the norm $\|u x\|_{u E}=$ $\|x\|_{E}, x \in E$. Assume throughout that such a diagonal transform of $E$ has the indicated norm. Of course, $u$ then acts as an isometry from $E$ onto $u E$. Thus, the statement that $u E=F$ is equivalent to the statement that $u$ is an isometry of $E$ onto $F$. It is easy to check that if $\varphi$ is dense in $E$, then $\varphi$ is dense in $u E$ and $(u E)^{f}=$ $(1 / u) E^{f}$, where $1 / u$ is given by $(1 / u)(s)=1 / u(s)$. Also routine is the equation $u(E \otimes F)=E \otimes(u F)$. 
Now let $E$ be a Banach space of functions on $S, W \subset S$, and let $K$ be the annihilator of $S \backslash W$ in $E$. Then $E$ will be said to have the factorization property relative to $W$ if there exists $\mathrm{b} \in M(E)$ such that $b \mid W$ acts as an isometry of $E \mid W$ onto $K \mid W$, i.e., $(b \mid W) E|W=K| W$.

Lemma 4. Let $E$ be a $B K$ space on $S, \varphi$ dense in $E, W \subset S$, and assume that $\varphi / W$ and $\varphi \mid S \backslash W$ are dense in $(S \backslash W)^{\perp} \mid W$ and $W^{\perp} \mid S \backslash W$, respectively, the annihilators taken in $E$. Then the factorization properties relative to $W$ for $E$ and $E^{f}$ are equivalent using the same factoring function.

Proof. If $b$ is the factoring function in either case, then $b$ vanishes at no point of $W$, since $\varphi \mid W$ is contained in both $K \mid W$ and $K^{\prime} \mid W$, where $K$ and $K^{\prime}$ are the annihilators of $S \backslash W$ in $E$ and $E^{f}$, respectively. Also, note that $M(E)=M\left(E^{f}\right)$.

Now $(b \mid W) E|W=K| W$ if and only if $((b \mid W) E \mid W)^{f}=(K \mid W)^{f}$. But by Lemma 1, $((b \mid W) E \mid W)^{f}=(1 / b \mid W)(E \mid W)^{f}=(1 / b \mid W) K^{\prime} \mid W$ and $(K \mid W)^{f}=E^{f} \mid W$. Therefore, $(b \mid W) E|W=K| W$ if and only if $(b \mid W) E^{f}\left|W=K^{\prime}\right| W$. This completes the proof.

THEOREM 2. Let $E$ be a $B K$ space on $S, W \subset S$. Assume that $E$ is series summable and that $E$ has the factorization property relative to $W$ with factoring function $b$. The following conditions are equivalent:

(i) $\mathscr{S}(E)$ has the factorization property relative to $W$ with factoring function b;

(ii) $M(E)$ has the factorization property relative to $W$ with factoring function b;

(iii) $E$ has the Nevanlinna-Pick property relative to $W$.

Proof. By [3], 6.5(b), the hypotheses of Lemma 4 are satisfied. Thus, (i) and (ii) are equivalent since $\mathscr{S}(E)^{f}=M(E)$.

The equivalence of (i) and (iii) follows from Theorem 1. To see this, note that condition (i) is equivalent to the requirement $(b \mid W) \mathscr{S}(E)\left|W=K^{\prime \prime}\right| W$ where $K^{\prime \prime}$ is the annihilator of $S \backslash W$ in $\mathscr{S}(E)$. Let $K^{\prime}$ be the annihilator of $S \backslash W$ in $E^{f}$. Now $(b \mid W) \mathscr{S}(E) \mid W=$ $(b \mid W)\left(E \otimes E^{f}\right)\left|W=(b \mid W)\left(E\left|W \otimes E^{f}\right| W\right)=E\right| W \otimes(b \mid W) E^{f} \mid W=$ $E\left|W \otimes K^{\prime}\right| W=\left(E \otimes K^{\prime}\right) \mid W$, using Lemma 2 twice. Therefore, condition (i) is equivalent to condition $K^{\prime \prime}\left|W=\left(E \otimes K^{\prime}\right)\right| W$. However, $K^{\prime \prime}$ and $E \otimes K^{\prime}$ vanish identically off $W$, so (i) is equivalent to $K^{\prime \prime}=E \otimes K^{\prime}$.

Under certain conditions the above results on $B K$ spaces may be applied to the Nevanlinna-Pick problem in more general Banach 
spaces of functions. The following results indicate this possibility.

If $E$ is a Banach space of functions, let $E^{2}$ be the linear span of $\{x y: x, y \in E\}$.

LEMma 5. Let $E$ be a Banach space of functions on $S, W \subset S$, $e \in E$, and assume that the annihilator of $W$ in $E^{2}$ is zero. Then $E$ has the Nevanlinna-Pick property relative to $W$.

Proof. Note that $E \subset E^{2}$ since $e \in E$, so the annihilator of $W$ in $E$ is also zero. For each $x \in E \mid W$ let $x^{\wedge}$ be the unique extension of $x$ to a member of $E$.

Let $u \in M(E \mid W)$ be arbitrary. Then $u \in E \mid W$ since $e \in E$. If $x \in E \mid W$, then $u^{\wedge} x^{\wedge} \in E^{2}$ and $(u x)^{\wedge} \in E \subset E^{2}$. Therefore, $u^{\wedge} x^{\wedge}=(u x)^{\wedge}$ since $u^{\wedge} x^{\wedge}$ and $(u x)^{\wedge}$ agree on $W$. It follows that $u^{\wedge} x^{\wedge} \in E$, so $u^{\wedge} \epsilon$ $M(E)$. Also, $\left\|u^{\wedge} x^{\wedge}\right\|_{E}=\left\|(u x)^{\wedge}\right\|_{E}=\|u x\|_{E \mid W}$, so $\left\|u^{\wedge}\right\|_{M(E)}=\|u\|_{M(E \mid W)}$. Therefore, $E$ has the Nevanlinna-Pick property relative to $W$.

LeMma 6. Let $E$ be a Banach space of functions on $S$. Assume that $E$ is a dual space with weak-star continuous point evaluations. Let $W_{n} \subset W_{n+1}$ for all $n$ and let $S=\bigcup_{n} W_{n}$. If $E \mid W_{n+1}$ has the Nevanlinna-Pick property relative to $W_{n}$ for all $n$, then $E$ has the Nevanlinna-Pick property relative to $W_{1}$.

Proof. Let $u^{1} \in M\left(E \mid W_{1}\right)$ and let $\varepsilon>0$ be given. Let $u^{2}$ be an extension of $u^{1}$ to $M\left(E \mid W_{2}\right)$ so that

$$
\left\|u^{2}\right\|_{M\left(E \mid W_{2}\right)} \leqq\left\|u^{1}\right\|_{M\left(E \mid W_{1}\right)}+\varepsilon / 2 \text {. }
$$

In general let $u^{n+1}$ be an extension of $u^{n}$ to $M\left(E \mid W_{n+1}\right)$ so that

$$
\left\|u^{n+1}\right\|_{M\left(E \mid W_{n+1}\right)} \leqq\left\|u^{n}\right\|_{M\left(E \mid W_{n}\right)}+\varepsilon / 2^{n} .
$$

For each $s \in S$ choose $m$ so that $s \in W_{m}$ and define $u(s)=u^{m}(s)$.

Let $\left\{s_{1}, s_{2}, \cdots, s_{n}\right\}$ be an arbitrary finite subset of $S$. Choose $m$ so that $\left\{s_{1}, s_{2}, \cdots, s_{n}\right\} \subset W_{m}$. Let $\pi^{s}$ be evaluation at $s$ for all $s \in S$. Consider $\sum_{i} c_{i} \pi^{s_{i}}$ as a member of $\left(E \mid W_{m}\right)^{*}$. Now

$$
\begin{aligned}
\left\|\sum_{i} u\left(s_{i}\right) c_{i} \pi^{s_{i}}\right\|_{E^{*}} & =\left\|\sum_{i} u^{m}\left(s_{i}\right) c_{i} \pi^{s_{i}}\right\|_{\left(E \mid W_{m}\right)^{*}} \\
& =\left\|\left(u^{m}\right)^{*}\left(\sum_{i} c_{i} \pi^{s_{i}}\right)\right\|_{\left(E \mid W_{m}\right)^{* *}} \\
& \leqq\left\|u^{m}\right\|_{M\left(E \mid W_{m}\right)}\left\|\sum_{i} c_{i} \pi^{s_{i}}\right\|_{\left(E \mid W_{m}\right)^{*}} \\
& \leqq\left(\left\|u^{1}\right\|_{M\left(E \mid W_{1}\right)}+\varepsilon\right)\left\|\sum_{i} c_{i} \pi^{s_{i}}\right\|_{E^{*}} .
\end{aligned}
$$

It follows from [8], Theorem 2, that $u \in M(E)$ and

$$
\|u\|_{M(E)} \leqq\left\|u^{1}\right\|_{M\left(E \mid W_{1}\right)}+\varepsilon
$$


Thus,

$$
\left\|u^{1}\right\|_{M(E) \mid W_{1}} \leqq\left\|u^{1}\right\|_{M\left(E \mid W_{1}\right)}
$$

The reverse inequality always holds. See [8], §2.

Consider the Hardy space $H^{p}$ and the Bergman space $A^{p}, 1 \leqq$ $p \leqq \infty$, as Banach spaces of functions on the unit disk $D=(|z|<1)$. For $p<\infty, H^{p}$ (or $A^{p}$ ) is the space of analytic functions $f$ on $D$ satisfying

$$
\begin{aligned}
& \|f\|_{p}=\sup _{0<r<1}\left(\frac{1}{2 \pi} \int_{-\pi}^{\pi}\left|f\left(\mathrm{re}^{i \theta}\right)\right|^{p} d \theta\right)^{1 / p}<\infty \\
& \text { (or } \left.\|f\|_{p}=\left(\frac{1}{\pi} \int_{D}|f|{ }^{p} d A\right)^{1 / p}<\infty\right) .
\end{aligned}
$$

$H^{\infty}$ is the space of bounded analytic functions on $D$ with $\|f\|_{\infty}=$ $\sup \{|f(z)|: z \in D\}$.

An elementary argument involving the restriction norm and factorization by Blaschke products yields the following result.

LEMMA 7. Let $S$ be a subset of $D, W \subset S, S \backslash W$ countable, and $\sum_{S \backslash W}(1-|z|)<\infty$. Then $E=H^{p} \mid S$ has the factorization property relative to $W$.

The Nevanlinna-Pick property was demonstrated for the Hardy spaces in [8], Corollaries 2 and 3 of Theorem 4. Using some of the same techniques one is able to achieve the Hardy space NevanlinnaPick property as a special case of the present work.

Theorem 3. For $1 \leqq p<\infty, H^{p}$ has the Nevanlinna-Pick property relative to every subset of $D$.

Proof. Assume first that $W \subset D$ satisfies $\sum_{W}(1-|z|)=\infty$. The annihilator of $W$ in $\left(H^{p}\right)^{2}$ is zero, since $\left(H^{p}\right)^{2}$ is contained in the Nevanlinna class. (See [1], p. 29, Exercise 1 and p. 18, Corollary). Therefore, by Lemma $5, H^{p}$ has the Nevanlinna-Pick property relative to $W$.

According to Lemma 6 , it suffices to prove for instance that $E=H^{p} \mid S$ has the Nevanlinna-Pick property relative to $W \subset S$, assuming that $\sum_{s}(1-|z|)<\infty$. The elementary properties of Blaschke products show that $E$ is strongly series summable. By Lemma $7, E$ has factorization property relative to $W$, so the hypotheses of Theorem 2 are satisfied. It now suffices to show that $\mathscr{S}(E)$ has the factorization property relative to $W$. 
By [8], Theorem $4, E^{f}$ is a diagonal transform of $H^{q} \mid S$ where $1 / p+1 / q=1$. The Hardy space factorization theory shows that $\mathscr{S}(E)$ is a diagonal transform of $H^{1} \mid S$. Using Lemma 7 again and the fact that diagonal transforms preserve the factorization property, one obtains the factorization property of $\mathscr{S}(E)$ relative to $W$. The result follows from Theorem 2 .

4. Applications to zero set and universal interpolating set problems. Let $E$ be a Banach space of functions on $S$. A proper subset $W$ of $S$ is called an $E$ zero set if there exists $f \in E$ such that $W=$ $\{s: f(s)=0\}$. For $W \subset S, E$ will be said to have the multiplier extension property relative to $W$ if $u \in M(E \mid W)$ implies there exists $v \in M(E)$ such that $v \mid W=u$. (The isometric part of the NevanlinnaPick property is being dropped.) For the present applications this weaker version of the Nevanlinna-Pick property is sufficient.

Observe that proper finite unions of $M(E)$ zero sets are $M(E)$ zero sets, since $M(E)$ is an algebra. Also, a proper union of an $E$ zero set and an $M(E)$ zero set is an $E$ zero set.

\section{TheOREM 4. Assume that}

(i) Finite subsets of $S$ are $M(E)$ zero sets; for instance, $M(E)$ contains a function which is one-to-one on $S$;

(ii) $E$ has the multiplier extension property relative to $E$ zero sets.

Then each $E$ zero set is contained in an $M(E)$ zero set.

Proof. Let $Z$ be an $E$ zero set, $s_{0} \in S \backslash Z, Z_{1}=Z \cup\left\{s_{0}\right\}$. Define a function $u$ on $Z_{1}$ by $u\left(s_{0}\right)=1, u(s)=0$ for $s \neq s_{0}$. Then $u \in E \mid Z_{1}$ since $Z$ is an $E$ zero set. Let $f \in E \mid Z_{1}$ be arbitrary. Then $u f=$ $f\left(s_{0}\right) u \in E \mid Z_{1}$, so $u \in M\left(E \mid Z_{1}\right)$. If $Z_{1}=S$ then $Z$ is an $M(E)$ zero set. If $Z_{1} \neq S$ then $Z_{1}$ is an $E$ zero set, since by (i), $\left\{s_{0}\right\}$ is an $M(E)$ zero set. Using (ii), $u \in M(E) \mid Z_{1}$. Therefore, $Z \subset\left\{s: u^{\wedge}(s)=0\right\}$, an $M(E)$ zero set, where $u^{\wedge}$ is an extension of $u$ to a member of $M(E)$.

CoROLLARY. Let $Z \subset D=(|z|)<1)$ be an $A^{p}$ zero set which is not an $H^{\infty}$ zero set. Then $A^{p}$ fails to have the multiplier extension property relative to $Z$. Hence, the Nevanlinna-Pick theorem fails for the Bergman spaces.

Proof. Since $M\left(A^{p}\right)=H^{\infty}$, condition (i) of Theorem 4 is satisfied. However, $Z$ cannot be contained in an $H^{\infty}$ zero set. Therefore, condition (ii) of Theorem 4 must be violated. It is well known that such sets $Z$ exist. 
Let $E$ be a Banach space of functions on $S, W \subset S$. Then $W$ will be called a universal interpolating set for $E$ if $M(E \mid W)=$ $l^{\infty}(W)$, the family of all bounded complex-valued functions on $W$.

THEOREM 5. Let $E$ be a Banach space of functions on $S, W \subset S$. Consider the following conditions on $E$ :

(i ) $M(E) \mid W=l^{\infty}(W)$;

(ii) $W$ is a universal interpolating set for $M(E)$;

(iii) $W$ is a universal interpolating set for $E$.

Then (i) and (ii) are equivalent, and each implies (iii). Furthermore, if $E$ has the multiplier extension property relative to $W$, then the three conditions are equivalent.

Proof. $M(M(E) \mid W)=l^{\infty}(W)$ if and only if $M(E) \mid W=l^{\infty}(W)$, since $e \in M(E) \mid W$. Also, $M(E) \mid W \subset M(E \mid W)$ and equality holds under the multiplier extension property.

In particular our definition of universal interpolating set coincides with the usual definition ([1], p. 147) in the case $E=H^{p}$.

THEOREM 6. Let $E$ be a Banach space of functions on $S$, and let $W$ be a subset of $S$ such that $\varphi \mid W$ is dense in $E \mid W$. Then $W$ is a universal interpolating set for $E$ if and only if $\left\{e^{s}: s \in W\right\}$ is an unconditional basis for $E \mid W$.

Proof. By definition, $\left\{e^{s}: s \in W\right\}$ is an unconditional basis for $E \mid W$ if and only if for all $x \in E \mid W$ the series $\sum_{W} x(s) e^{s}$ converges unconditionally to $x$ in $E \mid W$. As in [7], Theorem 5.1, $M(E \mid W)=$ $l^{\infty}(W)$ if and only if $\left\{e^{s}: s \in W\right\}$ is an unconditional basis for $E \mid W$, using [2], Theorem 4, Corollary 1.

The referee has kindly pointed out that the following is essentially a theorem of G. Köthe and O. Toeplitz. See [6], p. 529, Theorem 18.1.

THEOREM 7. Let $H$ be a Hilbert space of functions on $S$ with $\varphi$ dense in $H$. Then $S$ is a universal interpolating set for $H$ if and only if $H$ is a diagonal transform of $l^{2}(S)$.

Proof. Let $g(s)=\left\|e^{s}\right\|_{H}$ for all $s \in S$, and let $E=g H$. Then $\left\|e^{s}\right\|_{E}=1$. Also, $S$ is a universal interpolating set for $H$ if and only if $S$ is a universal interpolating set for $E$. Therefore, we may assume that $\left\|e^{s}\right\|_{H}=1$ for all $s \in S$.

Assume that $S$ is a universal interpolating set for $H$. Let $f_{1}=$ 
$\sum_{k=1}^{n} c_{k} e^{s_{k}} \in \varphi$ be given. Choose $u_{1}, u_{2}, \cdots, u_{n}$ as follows. Let $u_{1}=1$. Assume $u_{k}$ has been chosen. Choose $u_{k+1}$ with $\left|u_{k+1}\right|=1$ so that

$$
\operatorname{Re}<\sum_{i=1}^{k} u_{i} c_{i} e^{s_{i}}, u_{k+1} c_{k+1} e^{s_{k}}>_{H}=0 .
$$

Define $h$ on $S$ by $h\left(s_{k}\right)=u_{k}$ for $k=1,2, \cdots, n$ and $h(s)=1$ for all other $s \in S$. Then

$$
\left\|h f_{1}\right\|_{H}=\left\|f_{1}\right\|_{2} \text {. }
$$

Also, $h, 1 / h \in M(H)=l^{\infty}(S)$, so there exists a constant $K$ independent of $f_{1}$ so that

$$
\|h\|_{M(H)} \leqq K\|h\|_{\infty}=K
$$

and

$$
\|1 / h\|_{M(H)}<K
$$

Therefore,

$$
\left\|f_{1}\right\|_{2}=\left\|h f_{1}\right\|_{H} \leqq K\left\|f_{1}\right\|_{H}
$$

and

$$
\left\|f_{1}\right\|_{H}=\left\|(1 / h) h f_{1}\right\|_{H} \leqq K\left\|h f_{1}\right\|_{H}=K\left\|f_{1}\right\|_{2} .
$$

Since $\varphi$ is dense in $H$, it follows that $H=l^{2}(S)$.

The converse is obvious.

Theorem 7 shows that the above definition for universal interpolating sets in Banach spaces of functions is equivalent to the usual definition in the setting of Hilbert space. (See §1.)

CoRollary 1. Let $H$ be a Hilbert space of functions on $S$, $W \cong S$. For each $w \in W$ let $\pi^{w}(f)=f(w), f \in H$, and let $u(w)=1 /\left\|\pi^{w}\right\|$. Assume that $H$ has the multiplier extension property relative to $W$, and that $\varphi$ is dense in $H \mid W$. Then $W$ is a universal interpolating set for $M(H)$ if and only if $u H \mid W=l^{2}(W)$.

Proof. Let $E=(1 / u)(H \mid W)^{f}=(u H \mid W)^{f}$. Then $\left\|e^{w}\right\|_{E}=1$ for each $w \in W$. By hypothesis, $M(H \mid W)=M(H) \mid W$. Therefore, by Theorem 7, $W$ is a universal interpolating set for $M(H)$ if and only if $H \mid W$ is a diagonal transform of $l^{2}(W)$. But the latter condition is equivalent to $(u H \mid W)^{f}=E=l^{2}(W)$, i.e., $u H \mid W=l^{2}(W)$.

CoRollary 2. (Shapiro-Shields). Let $W=\left\{z_{n}\right\}$ be a sequence of points in the unit disk $D$, and let $u\left(z_{n}\right)=\left(1-\left|z_{n}\right|^{2}\right)^{1 / 2}$ for each $n$. 
Then $W$ is a universal interpolating set for $H^{\infty}$ if and only if $u H^{2} \mid W=l^{2}(W)$.

Proof. An easy calculation shows that $\left\|\pi^{z_{n}}\right\|_{2}=1 / u\left(z_{n}\right)$ for each n. Also, by Theorem 3, $M\left(H^{2} \mid W\right)=H^{\infty} \mid W$.

Of course, Shapiro and Shields were more interested in proving this kind of result using only the condition of Carleson which characterizes universal interpolating sets for $H^{\infty}$, thereby obtaining a simpler proof of Carleson's characterization. (See [5].)

THEOREM 8. Let $E$ and $F$ be Banach spaces of functions on $S$, and let $W \subset S$. Assume that $M(E) \subset M(F)$ and that $E$ has the multiplier extension property relative to $W$. If $W$ is a universal interpolating set for $E$, then $W$ is a universal interpolating set for $F$.

Proof. $M(F \mid W) \supset M(F)|W \supset M(E)| W=M(E \mid W)=l^{\infty}(W)$.

Corollary. Let $E$ be a Banach space of complex functions on the unit disk $D$ with $M(E) \supset H^{\infty}$. If $W \subset D$ is a universal interpolating set for $H^{\infty}$, then $W$ is a universal interpolating set for $E$.

The latter corollary generalizes part of [5], Theorem 4.

THEOREM 9. Let $H$ be a Hilbert space of functions on $S$ and assume $S$ contains an infinite subset $W$ with $\phi$ dense in $H \mid W$. Then $H$ has a universal interpolating sequence.

Proof. Let $W=\left\{z_{n}\right\}$ be a countable subset of $S$ with $\varphi$ dense in $H \mid W$. Let $E$ be the closure in $H$ of $\left\{e^{w}: w \in W\right\}$. As usual we may assume $\left\|e^{w}\right\|_{H}=1$ for all $w \in W$. Let $a_{n k}=\left\langle e^{z_{n}}, e^{z_{k}}\right\rangle_{H}$ for all $n, k$.

Now $E \mid W \supset l^{1}(W)$, so $(E \mid W)^{f} \subset l^{\infty}(W)$. It follows that $(E \mid W)^{f} \subset$ $c_{0}(W)$, the space of null functions on $W$. Therefore, $\lim _{k} a_{n k}=0$ for each $n$. Choose an increasing sequence $\left\{p_{n}\right\}$ of positive integers as follows. Let $p_{1}=1$. Having chosen $p_{n-1}$ choose $p_{n}>p_{n-1}$ so that

$$
\sum_{k=1}^{n-1}\left|a_{p_{k}, p_{n}}\right|<2^{-n}
$$

Then

$$
\begin{aligned}
\sum_{k \neq n}\left|a_{p_{k}, p_{n}}\right| & =\sum_{k=1}^{n-1}\left|a_{p_{k}, p_{n}}\right|+\sum_{k=n+1}^{\infty}\left|a_{p_{k}, p_{n}}\right|<2^{-n}+\sum_{k=n+1}^{\infty} 2^{-k} \\
& =2^{1-n} .
\end{aligned}
$$


Hence, for $n \geqq 1$ we have

$$
\sum_{k \neq n}\left|a_{p_{k}, p_{n}}\right|<\frac{1}{2} .
$$

A result of Schur [4] yields that for $x=\left\{x_{k}\right\}$,

$$
\left|\sum_{i \neq j} x_{i} \bar{x}_{j} a_{p_{i} \cdot p_{j}}\right| \leqq \frac{1}{2} \sum\left|x_{i}\right|^{2} \text {. }
$$

Therefore,

$$
\left\|\sum x_{i} e^{z} p_{i}\right\|_{H}^{2}=\sum\left|x_{i}\right|^{2}+\sum_{i \neq j} x_{i} \bar{x}_{j}\left\langle e^{\left.z p_{i}, e^{z} p_{j}\right\rangle_{H}}\right.
$$

But then

$$
\frac{1}{2} \sum\left|x_{i}\right|^{2} \leqq\left\|\sum x_{i} e^{z_{p_{i}}}\right\|_{H}^{2} \leqq \frac{3}{2} \sum\left|x_{i}\right|^{2}
$$

Let $W_{1}=\left\{z_{p_{i}}\right\}$, and let $F$ be the closure in $H$ of $\left\{e^{w}: w \in W_{1}\right\}$. It follows that $F \mid W_{1}=l^{2}\left(W_{1}\right)$.

Finally, using the first part of the proof choose a subset $W_{1}$ of $W$ such that $F \mid W_{1}$ is a diagonal transform of $l^{2}\left(W_{1}\right)$, where $F$ is the closure in $(H \mid W)^{f}$ of $\left\{e^{s}: s \in W_{1}\right\}$. It is easy to see that $\left(F \mid W_{1}\right)^{f}=$ $(H \mid W)^{f f} \mid W_{1}$. Also, $(H \mid W)^{f f}=H \mid W$ since $H \mid W$ is a Hilbert space with $\varphi$ dense. Therefore, $H \mid W_{1}$ is a diagonal transform of $l^{2}\left(W_{1}\right)$.

Not every Banach space of functions with $\phi$ dense has an infinite universal interpolating set.

Example. Let $E$ be the $B K$ space of sequences $x=\left\{x_{n}\right\}$ such that $\lim _{n} x_{n}=0$ and

$$
\|x\|=\left|x_{1}\right|+\sum_{n=1}^{\infty}\left|x_{n+1}-x_{n}\right|<\infty .
$$

For each increasing sequence $W=\left\{p_{n}\right\}$ of positive integers, $E \mid W$ is the set of functions $y$ on $W$ such that $\lim _{n} y\left(p_{n}\right)=0$ and

$$
\|y\|=\left|y\left(p_{1}\right)\right|+\sum_{n=1}^{\infty}\left|y\left(p_{n+1}\right)-y\left(p_{n}\right)\right|<\infty .
$$

Clearly, $M(E \mid W) \neq l^{\infty}(W)$, so $W$ is not a universal interpolating set.

\section{REFERENCES}

1. P. L. Duren, Theory of $H^{p}$ Spaces, Academic Press, 1970.

2. D. J. H. Garling, On topological sequence spaces, Proc. Cambridge Phil. Soc., 63 (1967), 997-1019. 
3. W. H. Ruckle, Representation and series summability of complete biorthogonal sequences, Pacific J. Math., 34 (1970), 511-528.

4. I. Schur, Bemerkungen zur Theorie der beschränkten Bilinearformen mit unendlich vielen Veränderlichen, J. für die Reine und Angewandte Math., 140 (1911), 1-28.

5. H. S. Shapiro and A. L. Shields, On some interpolation problems for analytic functions, Amer. J. Math., 83 (1961), 513-532.

6. I. Singer, Bases in Banach Spaces I, Springer-Verlag, 1970.

7. A. K. Snyder, Sequence spaces and interpolation problems for analytic functions, Studia Math., 39 (1971), 137-153.

8. Multipliers and the Nevanlinna-Pick theorem, Indiana Univ. Math. J., 25 (1976), 1159-1170.

Received August 23, 1977.

LEHIGH UNIVERSITY

BeTHLEHEM, PA 18015 



\section{PACIFIC JOURNAL OF MATHEMATICS}

\section{EDITORS}

RICHARD ARENS (Managing Editor)

University of California

Los Angeles, CA 90024

Charles W. Curtis

University of Oregon

Eugene, OR 97403

C. C. MOORE

University of California

Berkeley, CA 94720

\section{J. DugundJI}

Department of Mathematics

University of Southern California

Los Angeles, CA 90007

R. FinN and J. Milgram

Stanford University

Stanford, CA 94305

\section{ASSOCIATE EDITORS}
E. F. BECKENBACH
B. H. NeumanN
F. WOLF
K. YosHIDA

\section{SUPPORTING INSTITUTIONS}

\author{
UNIVERSITY OF BRITISH COLUMBIA \\ CALIFORNIA INSTITUTE OF TECHNOLOGY \\ UNIVERSITY OF CALIFORNIA \\ MONTANA STATE UNIVERSITY \\ UNIVERSITY OF NEVADA, RENO \\ NEW MEXICO STATE UNIVERSITY \\ OREGON STATE UNIVERSITY \\ UNIVERSITY OF OREGON
}

\author{
UNIVERSITY OF SOUTHERN CALIFORNIA \\ STANFORD UNIVERSITY \\ UNIVERSITY OF HAWAII \\ UNIVERSITY OF TOKYO \\ UNIVERSITY OF UTAH \\ WASHINGTON STATE UNIVERSITY \\ UNIVERSITY OF WASHINGTON
}

The Supporting Institutions listed above contribute to the cost of publication of this Journal, but they are not owners or publishers and have no responsibility for its content or policies.

Mathematical papers intended for publication in the Pacific Journal of Mathematics should be in typed form or offset-reproduced, (not dittoed), double spaced with large margins. Please do not use built up fractions in the text of the manuscript. However, you may use them in the displayed equations. Underline Greek letters in red, German in green, and script in blue. The first paragraph or two must be capable of being used separately as a synopsis of the entire paper. Items of the bibliography should not be cited there unless absolutely necessary, in which case they must be identified by author and journal, rather than by item number. Manuscripts, in triplicate, may be sent to any one of the editors. Please classify according to the scheme of Math. Reviews, Index to Vol. 39. All other communications should be addressed to the managing editor, or Elaine Barth, University of California, Los Angeles, California, 90024.

50 reprints to each author are provided free for each article, only if page charges have been substantially paid. Additional copies may be obtained at cost in multiples of 50 .

The Pacific Journal of Mathematics is issued monthly as of January 1966. Regular subscription rate: $\$ 72.00$ a year (6 Vols., 12 issues). Special rate: $\$ 36.00$ a year to individual members of supporting institutions.

Subscriptions, orders for numbers issued in the last three calendar years, and changes of address should be sent to Pacific Journal of Mathematics, 103 Highland Boulevard, Berkeley, California, 94708. Older back numbers obtainable from Kraus Periodicals Co., Route 100, Millwood, NY 10546.

PUBLISHED BY PACIFIC JOURNAL OF MATHEMATICS, A NON-PROFIT CORPORATION

Printed at Kokusai Bunken Insatsusha (International Academic Printing Co., Ltd.). 8-8, 3-chome, Takadanobaba, Shinjuku-ku, Tokyo 160, Japan.

Copyright (C) 1978 by Pacific Journal of Mathematics Manufactured and first issued in Japan 


\section{Pacific Journal of Mathematics}

\section{Vol. 76, No. $2 \quad$ December, 1978}

Stephanie Brewster Brewer Taylor Alexander, Local and global convexity in complete Riemannian manifolds ...........................

Claudi Alsina i Català, On countable products and algebraic convexifications of probabilistic metric spaces ...............................

Joel David Berman and George Grätzer, Uniform representations of

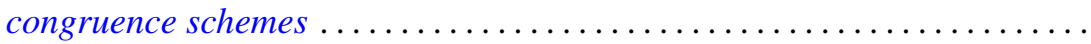

Ajit Kaur Chilana and Kenneth Allen Ross, Spectral synthesis in

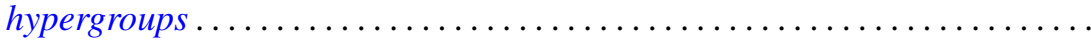

David Mordecai Cohen and Howard Leonard Resnikoff, Hermitian quadratic forms and Hermitian modular forms . .........................

Frank Rimi DeMeyer, Metabelian groups with an irreducible projective

representation of large degree .............................

Robert Ellis, The Furstenberg structure theorem .....................

Heinz W. Engl, Random fixed point theorems for multivalued mappings .......

William Andrew Ettling, On arc length sharpenings ..................

Kent Ralph Fuller and Joel K. Haack, Rings with quivers that are trees........

Kenneth R. Goodearl, Centers of regular self-injective rings ...............

John Gregory, Numerical algorithms for oscillation vectors of second order

differential equations including the Euler-Lagrange equation for

symmetric tridiagonal matrices.

Branko Grünbaum and Geoffrey Shephard, Isotoxal tilings

Myron Stanley Henry and Kenneth Leroy Wiggins, Applications of

approximation theory to differential equations with deviating

arguments

Mark Jungerman, The non-orientable genus of the n-cube .

Robert Richard Kallman, Only trivial Borel measures on $S_{\infty}$ are

quasi-invariant under automorphisms ................

Joyce Longman and Michael Rich, Scalar dependent algebras in the

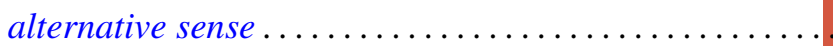

Richard A. Mollin, The Schur group of a field of characteristic zero ........ 471

David Pokrass, Some radical properties of rings with $(a, b, c)=(c, a, b) \ldots 479$

Margaret Shay and Paul Ruel Young, Characterizing the orders changed by

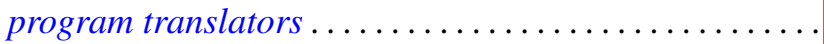

Jerrold Norman Siegel, On the structure of $B_{\infty}(F), F$ a stable space...

Surjeet Singh, (hnp)-rings over which every module admits a basic

submodule...

A. K. Snyder, Universal interpolating sets and the Nevanlinna-Pick property in

Banach spaces of functions...

Jeffrey D. Vaaler, On the metric theory of Diophantine approximation ... 